Proceedings of the 47th International School and Conference on the Physics of Semiconductors "Jaszowiec 2018"

\title{
Fourier-Transformed Temperature-Dependent Photoluminescence \\ of GaSb-Based Resonant Tunneling Structure with GaInAsSb Absorption Layer
}

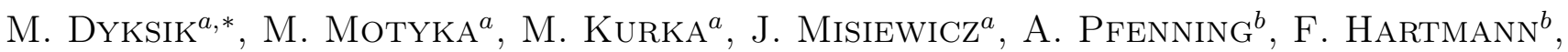 \\ R. WeIH ${ }^{b}$, L. WORSCHECH ${ }^{b}$, S. HÖFLING ${ }^{b, c}$ AND G. SĘK ${ }^{a}$ \\ ${ }^{a}$ Laboratory for Optical Spectroscopy of Nanostructures, \\ Department of Experimental Physics, Faculty of Fundamental Problems of Technology, \\ Wroclaw University of Science and Technology, Wybrzeże Wyspiańskiego 27, Wrocław 50-370, Poland \\ ${ }^{b}$ Technische Physik and Wilhelm Conrad Röntgen Research Center for Complex Material Systems, \\ Physikalisches Institut, Universität Würzburg, Am Hubland, D-97074, Würzburg, Germany \\ ${ }^{c}$ SUPA, School of Physics and Astronomy, University of St. Andrews, St. Andrews, KY16 9SS, United Kingdom
}

The band structure of type-II resonant tunneling structures with a quaternary GaInAsSb absorption layer was studied by means of Fourier-transformed photoluminescence in the mid-infrared spectral region. The temperatureresolved measurement revealed a low-energy band with non-trivial photoluminescence spectra evolution. At low temperature the emission originating from the confined states within the type II GaSb/AlSb/InAs quantum well dominates the spectrum. The increase of temperature triggers the hole transfer from the GaSb quantum wells to the heavy hole band edge of the quaternary GaInAsSb material. The GaInAsSb-related emission line arises at $130 \mathrm{~K}$ and gains in intensity up to room temperature.

DOI: 10.12693/APhysPolA.134.962

PACS/topics: GaSb-based resonant tunneling diodes, mid-infrared, photoluminescence

\section{Introduction}

Resonant tunneling structures (RTS) play an important role in a large variety of nowadays electronic and optoelectronic semiconductor devices. Besides being applied in high-speed electronic circuits, resonant tunneling is the basic underlying physical principle used in various other optoelectronic devices. Conventional RTS's based on $\mathrm{GaAs}$ or InP material systems are employed e.g. in active regions of quantum cascade lasers (QCLs) [1], THz oscillators $[2,3]$, and high-gain photodetectors $[4,5]$ with the possibility of single photon detection [6], as well as in nanothermometers [7].

Compared with conventional RTS's, the InAs-GaSbAlSb material system offers a broad spectrum of bandgap energies and band alignments from staggered (type-I) to broken (type-II) [8]. The latter is of particular interest as it provides possibility of covering the mid-infrared spectral range as an active region of interband cascade lasers (ICLs) and interband cascade detectors (ICD) [9]. Despite the advantage of flexibility for band gap engineering, the broken-gap alignment suffers from reduction of the oscillator strength, determining the gain of the medium. Substantial work was required, comprising the methods of optical spectroscopy, in order to understand

\footnotetext{
* corresponding author
}

the complex band structure of multilayered ICLs [10-12]. As a result of extensive studies, the ICLs exhibit a stateof-the-art performance in the spectral region of midinfrared [13].

On the other hand, there is very little information available on the exact energy structure of radiation detectors utilizing the type-II RTS. Such structures, with an Sb-based tunneling region [14, 15], are of great demand for high-speed and low-power requiring applications. The low power consumption arises because the negative resistance peak in the Sb-based material system occurs near $100 \mathrm{meV}$, whilst the conventional GaAs-based RTS generally requires the bias of several volts [8]. The Sb-RTS's also exhibit high peak current densities near $100 \mathrm{~A} / \mathrm{cm}^{2}$ that are desirable for high-speed applications, and large peak-to-valley current ratios as high as 20 at room temperature, that help to minimize power dissipation [8].

In this work, we present a comprehensive investigation of the energy band structure of the type-II RTS with an adjacent quaternary bulk layer. Similar material combination utilizing type-I GaAsSb/AlAsSb quantum wells (QW) with a GaInAsSb absorption layer was recently successfully reported as a photodetector operating in the mid-infrared spectral range [16, 17]. Due to the complicated band alignment of such systems in the vicinity of RTS's, as well as the possibility of interface layer formation, the structure under investigation exhibits non trivial photoluminescence (PL) behavior. The PL signal is measured in a wide range of sample's temperatures 
in order to determine the origin of constituent emission lines forming the emission spectrum. The analysis of the obtained data, together with theoretical simulations, provides the full picture of the band structure necessary for optimization of the fabrication of GaSb-based RTS's, which are expected to supplant the GaAs-based devices in terms of range of operating temperatures and power consumption.

\section{Investigated structures and experimental details}

The structure under investigation is a GaSb-based RTS grown by the molecular beam epitaxy. On top of a substrate, a $2 \times 10^{18} \mathrm{~cm}^{-3} p$-doped $300 \mathrm{~nm}$ thick GaSb bottom contact layer is deposited and followed by a $2 \times 10^{17}$ $\mathrm{cm}^{-3} p$-doped $30 \mathrm{~nm}$ thick GaSb transition layer and a $30 \mathrm{~nm}$ GaSb buffer layer. The active region contains of the RTS and a quaternary bulk-like absorption layer. The latter one is formed of $600 \mathrm{~nm}$ thick GaInAsSb. The In and As contents of $24 \%$ and $20 \%$, respectively, resulted in a negligible lattice mismatch with respect to GaSb. Finally, a $295 \mathrm{~nm}$ thick $2 \times 10^{18} \mathrm{~cm}^{-3} p$-doped GaSb top contact layer is grown. The structure is finalized with a $10 \mathrm{~nm}$ InAs cap. The design of the resonant part is constituted by an AlSb/InAs/GaSb/AlSb/InAs/GaSb double barrier structure forming two asymmetric InAs and one GaSb QWs. The width of consecutive layers equals to $2 / 1.5 / 3 / 2 / 2.5 / 5 \mathrm{~nm}$, respectively. The band alignment of the RTS, including $\mathrm{a} \approx 4 \mathrm{~nm}$ fraction of the $600 \mathrm{~nm}$ quaternary absorption layer, is presented in Fig. 1.

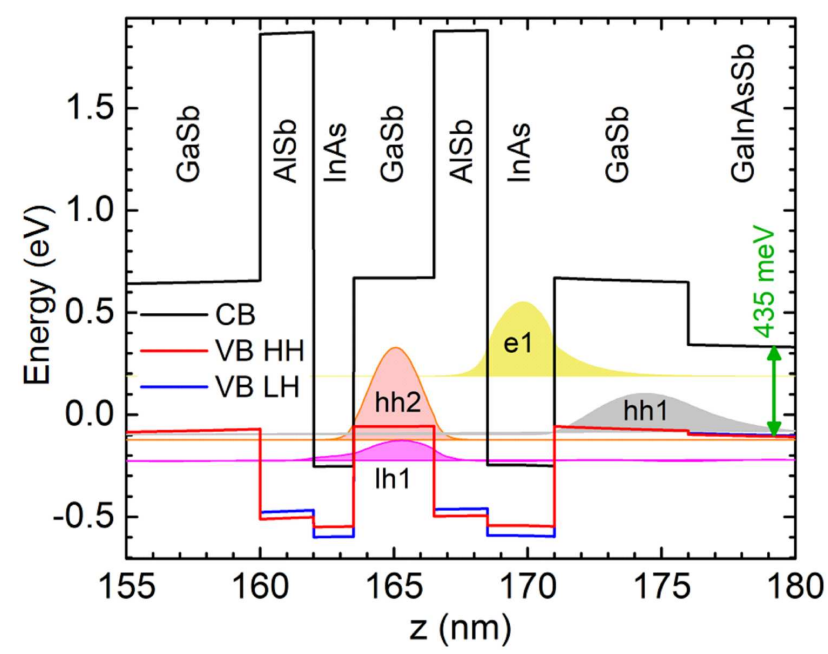

Fig. 1. Band alignment of the resonant tunneling part with a fraction of the GaInAsSb absorption layer. The conduction, heavy hole and light hole band edges are presented in black, red and blue, respectively. Colored areas indicate the probabilities for electron (e1) and holes (hh1, hh2 and lh1).

Photoluminescence studies in the spectral region of mid-infrared are performed with a Fourier transform infrared spectrometer operating in the step-scan mode.
The phase sensitive detection scheme utilizes a lock-in amplifier. The pump beam is mechanically chopped at a reference frequency of $360 \mathrm{~Hz}$ and focused onto the sample to the spot of $1 \mathrm{~mm}^{2}$. The spectra are measured with a spectral resolution of $64 \mathrm{~cm}^{-1}$, and the signal on the detector is averaged over time in order to improve the signal-to-noise ratio. To realize that, a standard procedure is employed where the spectrometer's mirror is fixed at each position for a time of $500 \mathrm{~ms}$. The temperatureresolved photoluminescence studies are performed with a continuous-flow liquid helium cryostat equipped with $\mathrm{CaF}_{2}$ windows.

\section{Results and discussion}

Figure 2 presents the evolution of the PL spectra in a wide temperature range from 15 to $300 \mathrm{~K}$. Two emission bands are visible: the low-energy one spreading from 300 to $500 \mathrm{meV}$ and a high-energy one, at about $780 \mathrm{meV}$. The former contains five distinct maxima labeled $I_{0}-I_{4}$, respectively. At low temperature (the topmost spectrum) the $I_{4}$ dominates, whereas the $I_{0}$ is the least visible feature. Both emission peaks vanish with temperature, the $I_{0}$ at $100 \mathrm{~K}$ and the $I_{4}$ at $75 \mathrm{~K}$. On the other hand, the emission peaks labeled $I_{1}, I_{2}$ and $I_{5}$ persist up to the room temperature. At elevated temperature of $180 \mathrm{~K}$ the $I_{3}$ peak emerges and dominates the PL spectrum at room temperature. The $I_{5}$ emission peak originates from the GaSb substrate.

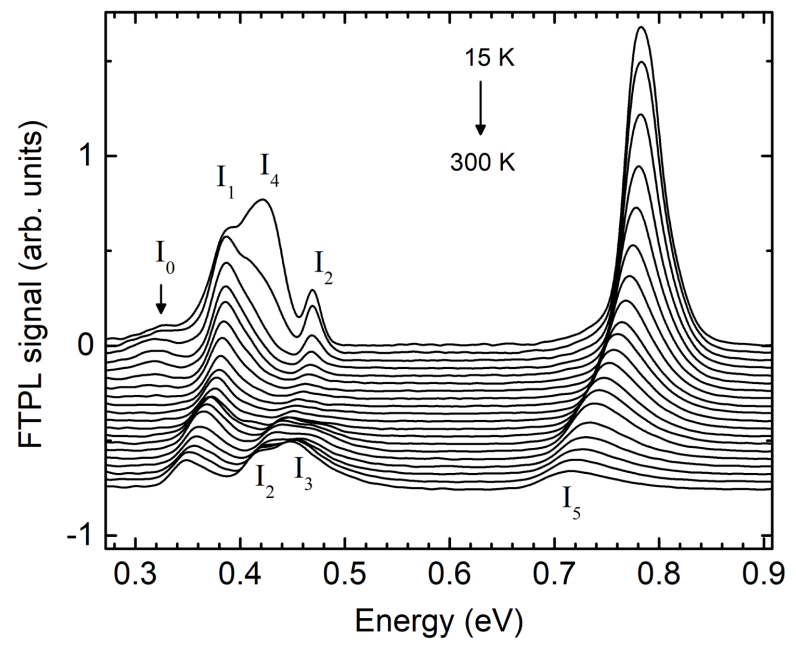

Fig. 2. PL spectra measured in a wide range of temperatures from 15 to $300 \mathrm{~K}$.

In order to determine the emission parameters of the $I_{0}-I_{5}$ peaks, each spectrum is fitted with the respective number of Gaussian functions. Figure 3a shows the decomposition of PL spectrum measured at low temperature $(30 \mathrm{~K})$ with five Gaussian peaks (colored area) standing for the $I_{0}, I_{1}, I_{2}, I_{4}$ and $I_{5}$ emission lines. A red dotted line stands for the cumulative fit. The asymmetry of the $I_{1}$ emission peak at the high energy side originates from the additional emission line labeled $I_{4}$. The 
room temperature $\mathrm{PL}$ response is presented in Fig. 3b. The emission peaks are broadened and the thermal highenergy tail is present (i.e. see peak labeled $I_{5}$ ). Due to the contribution of hot carriers the spectra are fitted with exponentially modified Gaussian function - a convolution of an exponential decay originating from the $k_{B} T$ tail and the symmetric Gaussian function. The cumulative fit (red dashed curve) shows good agreement with measured data.

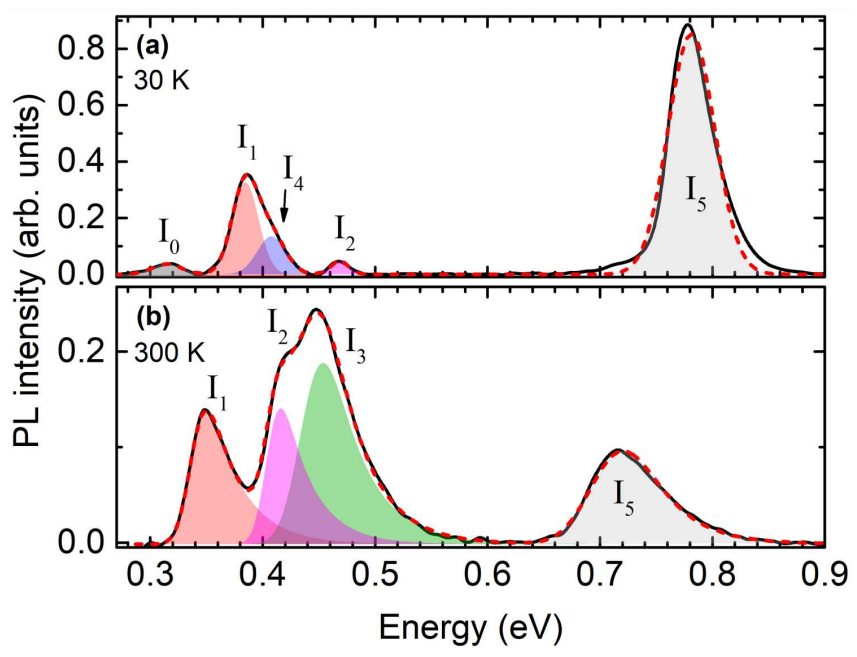

Fig. 3. The PL spectrum at $30 \mathrm{~K}$ (a) and $300 \mathrm{~K}$ (b). Colored areas represent the fitting results of individual emission lines. Red dotted line stands for cumulative fit.

In order to understand the origin of the $I_{0}-I_{4}$ emission lines the band structure was modeled in the framework of an 8-band $k p$ model with the commercial nextnano software [18]. Figure 1 shows the band alignment of the resonant-tunneling section of the structure under investigation, containing type-II AlSb/InAs/GaSb QW, as well as GaSb buffer layers and a fraction of the $600 \mathrm{~nm}$ wide quaternary GaInAsSb absorption layer. The confined states of electrons and holes and the respective probability densities are calculated. The ground electron state is localized mainly in the $2.5 \mathrm{~nm}$ wide InAs QW. The holes of interest (i.e. with substantial squared overlap integral with the e1 state) reside in two subbands. The ground state (hh1 in grey) is located in a shallow, $5 \mathrm{~nm}$ wide GaSb QW, with InAs and GaInAsSb heavy hole band edges creating a confining potential of $10 \mathrm{meV}$. The second subband contains the hh2 and lh1 states, in red and magenta respectively, and is located in the $3 \mathrm{~nm}$ wide GaSb QW.

The calculated values (dashed lines) of transition energies are compared with the ones obtained from the fitting procedure in Fig. 4a. According to the band structure simulations, the $\mathrm{I}_{1}$ emission line (red diamonds) corresponds to the e1-hh2 optical transition in the type-II InAs/GaSb quantum well, whereas the origin of $\mathrm{I}_{2}$ emission peak is still under investigation. The possible explanations are the e1-lh1 transition (magenta) within the same QW and/or the transition involving the light hole subband of the quaternary GaInAsSb layer. The $\mathrm{I}_{1}$ and $\mathrm{I}_{2}$ transitions are visible in the whole range of the measurements temperature. At low temperature (see Fig. 3a) the $I_{0}$ emission line is present at the low energy side of the spectrum corresponding to the e1-hh1 transition. The $\mathrm{I}_{3}$ emission peak, dominating the $\mathrm{PL}$ spectrum at room temperature (see Fig. 3b), originates from the quaternary $\mathrm{Ga}_{0.76} \mathrm{In}_{0.24} \mathrm{As}_{0.2} \mathrm{Sb}_{0.8}$ bulk layer.

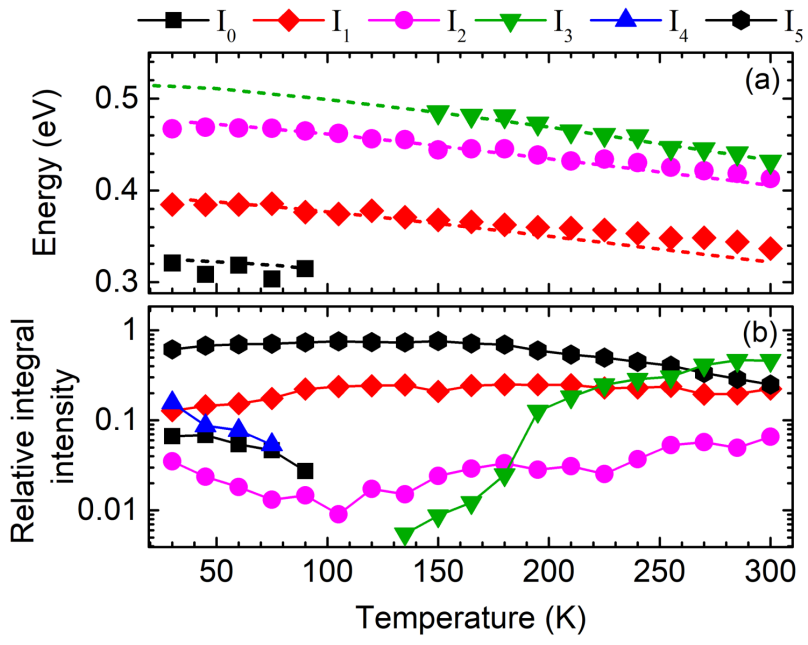

Fig. 4. (a) The transition energies extracted from fits to the PL spectra in function of sample temperature. Dashed curves represent the results of computer simulations. (b) Relative integral intensities as a function of sample's temperature.

Figure $4 \mathrm{~b}$ presents the relative integral intensities defined as the ratio of the area of individual peak to the total area of the cumulative fit. The change of temperature from 15 to $300 \mathrm{~K}$ triggers the hole transfer within the sample. In the range of low temperatures, from 15 to $100 \mathrm{~K}$, the $I_{0}$ emission line exhibits an efficient thermal quenching due to the shallow confining potential for the hh1 state in the GaSb QW. The $I_{0}$ emission line disappears completely at $\approx 100 \mathrm{~K}$, which, translating to the thermal energy scale, gives $8.5 \mathrm{meV}$ activation energy a value reasonably close to the $\mathrm{QW}$ depth of $10 \mathrm{meV}$. As the holes from GaSb (from the GaSb QW as well as from the GaSb top contact layer) are thermally activated to the heavy hole band edge of GaInAsSb, the $I_{3}$ emission line, corresponding to the GaInAsSb material, arises at approximately $130 \mathrm{~K}$. At the temperatures above $150 \mathrm{~K}$, the intensity of the $I_{1}$ peak, attributed to the e1 to hh2 transition, remains nearly constant, whereas the $I_{2}$ emission line, representing the e1-lh transition, exhibits a rising trend. The origin of the $I_{4}$ emission line is still not fully clear yet, however the possible explanation is that it comes from the InAs cap layer.

\section{Conclusions}

We have investigated a GaSb-based type-II resonant tunneling structures with the GaInAsSb absorption layer by means of temperature-dependent photoluminescence 
supported by band structure modeling. The PL spectra revealed low-energy emission band containing five peaks, whose evolution was studied in function of temperature. At low temperature the emission originates mostly from the confined states in the type-II InAs/AlSb/GaSb quantum well within RTS and a shallow InAs/GaSb QW adjacent to the GaInAsSb layer. The confinement potential for the shallow GaSb QW was determined to be $8.5 \mathrm{meV}$, in agreement with the calculated confined levels' structure. At about $130 \mathrm{~K}$, the emission from the quaternary GaInAsSb bulk layer arises, dominating the photoluminescence spectrum at room temperature.

\section{Acknowledgments}

This research was carried out with financial support from the National Science Center of Poland within Grant No. $2016 / 21 / \mathrm{N} / \mathrm{ST} 7 / 02790$

\section{References}

[1] M. Dyksik, M. Motyka, W. Rudno-Rudziński, G. Sęk, J. Misiewicz, D. Pucicki, K. Kosiel, I. Sankowska, J. Kubacka-Traczyk, M. Bugajski, J. Infrared Millim. Terahertz Waves 37, 710-719 (2016).

[2] S. Suzuki, M. Asada, A. Teranishi, H. Sugiyama, H. Yokoyama, Appl. Phys. Lett. 97, 1 (2010).

[3] T. Maekawa, H. Kanaya, S. Suzuki, M. Asada, Appl. Phys. Express 9, 024101 (2016).

[4] A. Pfenning, F. Hartmann, F. Langer, S. Höfling, M. Kamp, L. Worschech, Appl. Phys. Lett. 104, 101109 (2014).

[5] A. Pfenning, F. Hartmann, F. Langer, M. Kamp, S. Höfling, L. Worschech, Nanotechnology 27, 355202 (2016).

[6] J.C. Blakesley, P. See, A.J. Shields, B.E. Kardynałl, P. Atkinson, I. Farrer, D.A. Ritchie, Phys. Rev. Lett. 94, 1 (2005).

[7] A. Pfenning, F. Hartmann, M. Rebello Sousa Dias, L.K.L.K. Castelano, C. Süßmeier, F. Langer, S. Höfling, M. Kamp, G.E.G.E. Marques, L. Worschech, V. Lopez-Richard, ACS Nano 9, 6271 (2015).
[8] B.R. Bennett, R. Magno, J.B. Boos, W. Kruppa, M.G. Ancona, Solid State Electron. 49, 1875 (2005).

[9] M. Motyka, K. Ryczko, G. Sęk, F. Janiak, J. Misiewicz, A. Bauer, S. Höfling, A. Forchel, Opt. Mater. (Amst.) 34, 1107 (2012).

[10] M. Dyksik, M. Motyka, M. Kurka, K. Ryczko, M. Dallner, S. Höfling, M. Kamp, G. Sęk, J. Misiewicz, Opt. Quantum Electron. 48, 401 (2016).

[11] M. Dyksik, M. Motyka, R. Weih, S. Höfling, M. Kamp, G. Sęk, J. Misiewicz, Opt. Quantum Electron. 49, 59 (2017).

[12] M. Motyka, K. Ryczko, M. Dyksik, G. Sęk, J. Misiewicz, R. Weih, M. Dallner, S. Höfling, M. Kamp, J. Appl. Phys. 117, 084312 (2015).

[13] I. Vurgaftman, R. Weih, M. Kamp, J.R. Meyer, C.L. Canedy, C.S. Kim, M. Kim, W.W. Bewley, C.D. Merritt, J. Abell, S. Höfling, J. Phys. D Appl. Phys. 48, 123001 (2015).

[14] A. Pfenning, G. Knebl, F. Hartmann, R. Weih, A. Bader, M. Emmerling, M. Kamp, S. Höfling, L. Worschech, Appl. Phys. Lett. 110, 033507 (2017).

[15] A. Pfenning, G. Knebl, F. Hartmann, R. Weih, M. Meyer, A. Bader, M. Emmerling, L. Worschech, S. Höfling, Appl. Phys. Lett. 111, 1 (2017).

[16] F. Rothmayr, A. Pfenning, C. Kistner, J. Koeth, G. Knebl, A. Schade, S. Krueger, L. Worschech, F. Hartmann, S. Höfling, Appl. Phys. Lett. 112, 161107 (2018).

[17] F. Hartmann, A. Pfenning, G. Knebl, R. Weih, A. Bader, M. Emmerling, M. Kamp, S. Höfling, L. Worschech, Proc. SPIE 10403, 10403-10403-7 (2017).

[18] S. Birner, S. Hackenbuchner, M. Sabathil, G. Zandler, J.A. Majewski, T. Andlauer, T. Zibold, R. Morschl, A. Trellakis, P. Vogl, Acta Phys. Pol. A 110, 111 (2006). 